\title{
Effective Cancer Management can only be Possible via a Multi- Disciplinary Team Approach - Need for Establishment of Site Specific Tumor Boards
}

\author{
Ahmed Nadeem Abbasi* \\ Department, Section of Radiation Oncology, Department of Oncology. The Aga Khan University, Karachi, Pakistan \\ doi.org/10.21089/njhs.11.0002
}

\section{INTRODUCTION}

In Pakistan there is no proper National Cancer Registry. The age-standardized rates (ASR) for cancer (all sites), 1995 to 1997 in Karachi South (KS) were 139.11/100000 in males and 169.5/100000 in females. Corresponding rates for 1998 to 2002 were $179.0 / 100000$ in males and 204.1/100000 in females. In the 1995-1997 data, the most common malignancies (ASR per 100,000) in males were lung (21.3), oral cavity (14.2), urinary bladder (9.0), and larynx (8.8). The commonest cancers in females were breast (53.1), oral cavity (14.5), and ovary (10.9). Data from 1998 to 2002 showed a rising incidence for lung (25.5), larynx (11.8), and urinary bladder (9.9) cancers in males and breast (69.1), esophagus (8.6), and cervical (8.6) cancers in females. The mean age of cancer for all sites was 51.2 years $(95 \%$ CI 49.4 ; $53.1)$ for males and 50.0 years $(95 \%$ CI $48.2 ; 52.4)$ for females in 1995-1997. Corresponding rates for 1998-2002 were 49.5 years $(95 \% \mathrm{CI} 47.5 ; 51.4)$ in males and 53.7 years (95\% CI51.5; 55.6) in females [1]. Some institutions are now starting to develop their own Cancer Registry.

In the United States, the estimated Cancer Cases reported in 2009 were about 1.5 million new cases, comprising of 766,130 men, and 713,220 women. These figures excluded basal and squamous cell skin cancers and in situ carcinomas except urinary bladder. Cancers of the prostate and breast will be the most frequently diagnosed cancers in men and women, respectively, followed by lung and colorectal cancers in both men and in women [2]. Cancer deaths reported by National Center for Health Statistics is 559,888, equating to $23.1 \%$ of all deaths [3].

\section{INTERNATIONAL CANCER SCENARIO}

The global cancer incidence estimate by the GLOBOCAN 2002 was $8.7 \%$ (men 13.3\%; women 3.4\%) under-estimated due to under-ascertainment in elderly cases, which means, worldwide, new cancer cases in 2002 were $11,810,000$ $(6,574,000 \mathrm{men} ; 5,236,000$ women), topping the original estimate by 1 million [4].

The crude annual incidence of cancer in Europe has been estimated at 338 per 100, 000 population for eastern Europe and 447 per 100000 population in western Europe [5]. Each year more than 284,000 people are diagnosed with cancer (excluding non-melanoma skin cancer) in the UK, and more than 1 in 3 people will develop some form of cancer during their lifetime [6]. In 2007, there were more than 155,000 cancer deaths in the UK, and one in four (27\%) of all deaths in the UK were due to cancer [7].

In Europe, on the other hand, the relevant information for 2008 is not generally available as yet, therefore, statistical models were used to estimate incidence and mortality data for 25 cancers in 40 European countries (grouped and individually) in 2008. The calculations were based on published data. If not collected, national rates were estimated from national mortality data and incidence and mortality data provided by local cancer registries of the same or neighboring country. There were an estimated 3.2 million new cases of cancer and 1.7 million deaths from cancer in 2008 [8].

\section{NEED FOR A PAKISTANI NATIONAL CANCER PLAN}

Despite the lack of Registry and population based data on demographics and characteristics of various cancers, this fact is being observed by practicing clinicians that now they are diagnosing more malignancies in their clinics than ever before. One of the typical prototype example is squamous cell carcinoma of Head and Neck which is found to be associated with the use of tobacco in the form of betel leaf, betel nuts, various forms of chewing tobaccos, gutkas and a combination of some or all of these harmful substances. 
As rising cancer incidence is a global issue, and some cancers are preventable, health policy makers took this issue as a national priority. Countries like USA, UK, France, and others had made their National Cancer Plans, and they are in the process of implementing their goals and objectives.

We in Pakistan are still struggling to develop our strategies. Being a developing country we cannot afford to provide state of the art treatment facilities to our patients. The overall expenses of cancer management are huge and lack of resources is a major factor, which plays an important role in the poor outcome of this disease in our country. Patients present late and by the time their tumours are diagnosed, they can only be considered for palliative treatment.

\section{MULTI-DISCIPLINARY APPROACH}

With the above mentioned suboptimal starting point, we are facing another issue of treatment. Other countries of the world had learnt the hard lessons and now they've realized that cancer management is not a one man show. It is of highest importance and relevance to involve key stake holders in the form of all concerned specialties before embarking on a comprehensive management plan for a particular patient, a continuous provision of good quality care and clinical services is only possible with multidisciplinary team work.

Being looked after by a multidisciplinary team, the patient's faith and confidence increases markedly just as an assurance of not being treated by a single clinician.

The UK Cancer Plan set out the first comprehensive national cancer programme for England. It had four aims, which were centered on tackling health inequalities in order to get down the death toll of the unskilled workers, who were twice as likely to die of cancer as compared to the professionals. They invested into strong cancer research and genetics, to save more lives, ensuring provision of the best available support, care and treatment to all the cancer patients. This plan provided a strategy for bringing together prevention, screening, diagnosis, treatment and care for cancer and the investment needed to deliver these services in terms of improved staffing, equipment, drugs, treatments and information systems [9].

Similarly, the National Comprehensive Cancer Network $(\mathrm{NCCN})$, a not-for profit alliance of 21 of the world's leading cancer centers, is dedicated to improving the quality and effectiveness of care provided to patients with cancer. As the arbiter of high-quality cancer care, NCCN promotes the importance of continuous quality improvement and recognizes the significance of creating clinical practice guidelines appropriate for use by patients, clinicians, and other health care decision-makers. NCCN Member Institutions pioneered the concept of the multidisciplinary team approach to patient care and lead the fight against cancer as they integrate programs in patient care, research, and education. Their programs offer access to expert physicians, superior treatment, and quality and safety initiatives that continuously improve the effectiveness and efficiency of cancer care [10].

There are many practicing Oncologists in Pakistan, who are serving our cancer patients with the best of their clinical knowledge and skills. There is a growing tendency amongst members of our oncology community to form Expert Panel Multi Disciplinary Tumour Boards, and to discuss all cases in this panel before offering the treatment to the patient. As this is a recommended international approach, specialists are quite willing to adopt this culture of sharing ideas with their colleagues. An evidence of multidisciplinary approaches providing optimal treatment outcome for locally advanced head and neck cancer, with overall survival in these patients being comparable to that reported in randomized clinical trials was reported by Nguyen NP et al. in their retrospective analysis of 213 patients treated for locally advanced head and neck cancer in a single institution [11]. This effective clinical approach will provide opportunities to individual members of the team to speak directly to each other about clinical matters, discussing the often forgotten clinical management protocols, thus enhancing their quality of care and outcome. Ideally, no cancer patient should be treated without making a comprehensive management plan which has to be agreed upon by consensus in an Expert Panel Multi-Disciplinary Tumour Board. In Pakistan, institutional site specific teams are in the early phase of establishment at the moment [12].

\section{ESTABLISHMENT OF SITE SPECIFIC MULTI- DISCIPLINARY TUMOUR BO-ARDS \& PEER REVIEWED PRACTICE}

A site specific Multi-Disciplinary Tumour Board can be initiated with core specialist members. The core constituents of a Tumour Board are Oncologists, Site Specific Surgeons, Radiologists, Pathologists and Physicians. Other members from the allied healthcare services like Nutritionists can also participate. Post - graduate trainee doctors can learn and develop valuable clinical skills while attending the Boards. This leads towards easy access of knowledge, where case discussion leads to maturation of ideas that may not have been apparent to a single treating clinician. Team working increases the sense of partnership and provides friendship and support particularly in difficult clinical situations such as the management of clinical errors and complaints.

Now, as we are witnessing a positive change, it is high time for all the Pakistani specialists involved in the care and management of cancer patients to come forward and establish these boards both in private and public sector hospitals, following the steps taken by the Health sector of United Kindgdom and Ireland. Hopefully we would go through the same flux, and after initial teething problems, we would be able to convince our non-oncology colleagues to 
come forward and help us in the formation of these team meetings, which can be conducted on a regular basis with a view of covering all newly diagnosed cases.

Peer reviewed clinical practice is recommended as an internationally acceptable quality standard and is an identified area of improvement for our specialized services.

\section{ACKNOWLEDGEMENT}

Declared none.

\section{CONFLICT OF INTEREST}

Declared none.

\section{REFERENCES}

[1] Bhurgri Y, Bhurgri A, Nishter S, Ahmed A, Usman A, Pervez S, et al. Pakistan--country profile of cancer and cancer control 19952004. J. Pak. Med. Assoc., 2006; 56(3): 124-30.

[2] Cancer Facts \& Figures 2009. Atlanta: American Cancer Society; 2009.

[3] US Mortality Data 2006, Centers for Disease Control and Prevention, 2009 [database on the Internet].
[4] Fallah M, Kharazmi E. Global cancer incidences are substantially under-estimated due to under-ascertainment in elderly cancer cases. Asian Pac. J. Cancer Prev., 2009; 10(2): 223-6.

[5] International Agency for Research on Cancer, The GLOBOCAN 2002 software and database, version 2.0, 2004 [database on the Internet]. [cited. Available from: http://www-dep.iarc.fr/.

[6] Quinn M BP, Kirby L, Brock A. Registrations of cancer diagnosed in 1994-1997, England \& Wales. Health. Statistics Quarterly 07 Autumn 2000: Office for National. Statistics, 2000: 71-82. 6.

[7] http://info.cancerresearchuk.org/cancerstats/index.htm.

[8] Ferlay J, Parkin DM, Steliarova-Foucher E. Estimates of cancer incidence and mortality in Europe. Eur. J. Cancer, 2008; 46(4): 765-81.

[9] Health Do. The NHS Cancer Plan: a plan for investment a plan for reform, Published date: 27 September 2000, Primary audience: Professionals, Gateway reference:not required, Product code: 22293, Copyright holder: Crown.

[10] www.nccn.org/ [database on the Internet].

[11] Nguyen NP, Vos P, Lee H, Borok TL, Karlsson U, Martinez T, et al. Impact of tumor board recommendations on treatment outcome for locally advanced head and neck cancer. Oncol., 2008; 75(3-4): 186-91.

[12] Abbasi AN, Cancer management is a multidisciplinary team work. J. Coll. Phys. Surg. Pak., 2011; 21(5): 259-61. doi: 05.2011/JCPSP.259261

Received: May 16, 2016

Copyright $@ 2016$ NJHS - All Rights Reserved

This is an open-access article. 